\title{
Search of Biomarker in the Oral Rehabilitation
}

\author{
Masakazu Azuma1,2, Senichi Suzuki1,2, Masaki Sawa², Tomoko Yoshizawa², Ailing Hu³, \\ Takuji Yamaguchi ${ }^{3}$, Hiroyuki Kobayashi ${ }^{2,3}$ \\ ${ }^{1}$ Lion Implant Center, Ebina-shi, Japan \\ ${ }^{2}$ Department of Hospital Administration, Juntendo University Graduate School of Medicine, Bunkyo-ku, Japan \\ ${ }^{3}$ Center of Advanced Kampo Medicine and Clinical Research, Juntendo Graduate School of Medicine, \\ Bunkyo-ku, Japan \\ Email: azuma-dental@nifty.com
}

Received 14 December 2015; accepted 22 January 2016; published 25 January 2016

Copyright (C) 2016 by authors and Scientific Research Publishing Inc.

This work is licensed under the Creative Commons Attribution International License (CC BY). http://creativecommons.org/licenses/by/4.0/

(c) (i) Open Access

\section{Abstract}

Are there any ways to analyze objectively if any good changes happen to the bodies for the patients with acquired edentulous by getting a treatment to recover occlusion? In this study, we focused on interleukin 6 (IL-6) as inflammatory cytokine, cortisol known as a stress related substance and secretory immune globulin A (SIgA) related to immune reaction, and chose 14 patients who had occlusion reconstructed by the immediately loaded dental implants because occlusion contact with maxillary and mandibular dentition was lost caused by edentulous jaws or maxillary and mandibular teeth crossing each other and their jaw position and central occlusion position could not settle, and verified the relationship with changes of patients' physical and mental states during the 6 months of the treatment and the effect of the immediately loaded implants treatment by using saliva which was non-invasive and easy to sample in biomarkers in saliva. Moreover for female subjects, the changes of facial color tones were measured by using Robo Skin Analyzer ${ }^{\circledR}$, a digital image analyzer, to measure the condition of their skin. In conclusion, the positive correlation between the amount of IL- 6 and cortisol was not admitted. However, it was admitted that IL-6 tended to increase when a systemic change which interrupted curing such as the interruption of fusion of the implant and the bone was observed even though the patient did not notice any particular symptoms and cortisol tended to increase when the patient noticed discomfort and mentioned any events under stress on his or her medical record. Moreover with the skin color analysis by Robo Skin Analyzer ${ }^{\circledR}$, the possibility that the occlusion treatment by the immediate implant had an effect on physical and psychological health promotion through the acquisition of the masticatory function and the aesthetic recovery was indicated.

\section{Keywords}

Occlusion, Immediate Load Dental Implants, IL-6, Cortisol, Skin Condition 


\section{Introduction}

Recently, in the choices of prosthesis treatments for patients with edentulous to recover the masticatory function and the sensuousness, the recovery of occlusional function (oral rehabilitation) applying dental implant is becoming common besides the treatment with removal dentures which has been the mainstay for a long time. The reconstruction of occlusion with implant fixture which made it possible to recover the function disorder caused by the deficit of a tooth and a jawbone and the morphological disorder which were once considered difficult to do with a removal denture because it loaded the oral mucosa absorbability instantly became the focus of the research and the development as a new trend of dental care, and grew to the reliable treatment method keeping its technical stability. On the other hand, there are several problems regarding patients' physical and psychological burdens, such as costs and surgical stress, and especially to shorten the duration of treatment which is required until the occlusion function recovers has been expected. It makes the immediately loaded implants by All-on-4 reality to meet the patients' desire [1], "I want occlusion get recovered immediately". The concept of the immediate load dental implants [2] and All-on-4 taking full advantage of its characteristic feature is not to regenerate the lost paradentium biologically but to fix strongly the implant superstructure consists of the artificial tooth and the artificial substitute for paradentium, and the supporter composed of 4 implants fixtures implanted in the jawbone to the residual ridge after the tooth loss through the attachment of screws. After the tooth loss, only four implant bodies are placed to the staunch part on the remaining jawbone keeping the ideal depth by making an angle to the implant direction to gain stronger fusion. Furthermore, geometric resistance can be obtained by arranging the four placed implant fixtures evenly like making a horseshoe arch from occlusal surface.

This resistance does not only avoid the each patient's unique habitual lateral movement of jaw causing fibrous healing which blocks the fusion of the implant fixtures and the bone and the tiny movement caused by traumatic occlusal force, but also obtains the strength against occlusion. This uniqueness of All-on-4 makes the immediate functional loading which reconstructs occlusion by dentition with 12 artificial teeth for both upper and low jaws possible for edentulous patients just after the dental implant surgery.

In this study, we targeted patients with a provisional bridge which was the therapeutic upper structure applied for six months until the final form of the implant super structure was completed, focused on interleukin 6 (IL-6), cortisol and SIgA in saliva and verified the relationship with the effect of obtaining the occlusional function and the changes of patients' physical and psychological states during the immediate load dental implant treatment. Moreover for female subjects, their skin colors were measured with the color information from the digital image of the skin taken by a CCD camera using Robo Skin Analyzer ${ }^{\circledR}$, a digital image analyzer, and measured the changes of their facial skin tones.

\section{Material and Method}

\subsection{Subject}

The subjects were 14 patients (64.1 \pm 5.8 years), 7 males (68.7 \pm 4.5 years) and 7 females (59.4 \pm 2.1 years), who visited Ebina Lion Implant Center with complaints that the contact with the maxillary and mandibular dentition was lost because of edentulous jaws or maxillary and mandibular teeth crossing each other and their mandibular positions could not settle and requested for occlusion recovery with immediate load dental implants to July 2014 from January 2013. The subjects were obtained consent by a purpose of this study and this research was performed according to the ethic official regulation that nonprofit foundation Japanese Society of Oral Implantology to belong to of the Ebina Lion Implant Center established.

Patients who received a diagnosis when in condition not to be able to take the centric occlusion for completely losing occlusal contact were chosen as a subject. After obtaining consent by a purpose of this study, they started taking saliva before and right after the implant placement surgery and at the monthly follow-ups after the surgery. The subjects were placed a provisional bridge as the upper structure right after the implant placement surgery, and occlusion of the upper and lower jaws were recovered. Replace Select Tapered $\mathrm{RP}^{\circledR}$ (Nobel Biocare Japan K.K.) was used as the implant fixture, and tightening torque over $35 \mathrm{~N} \mathrm{~cm}$ for all of the placed implant fixtures were confirmed. The temporary bridge was composed with the resin artificial tooth and dental polymerized resin, and Temporary Cylinder Plastic ${ }^{\circledR}$ (DCA468-0) and Prosthetic Screw ${ }^{\circledR}$ (29285) (Nobel Biocare Japan K.K.) were used as its connector. 


\subsection{The Method for Collecting Saliva}

As for collecting saliva for the test, the duration was determined for six months which was required for ISQ, the bone fusion indicator that implant prosthetics recommend, to get over 70 until paradentium stabilized and the provisional bridge transited to the final prosthesis [3] [4]. Interleukin 6 (IL-6) as inflammatory cytokine, cortisol known as a stress related substance and secretory immune globulin A (SIgA) were measured.

After putting bland and innocuous Salimetrics Oral Swab (Salimetrics, USA) into the subject's mouth for 5 minutes in a sitting posture, it was collected and the saliva was taken by centrifugation (1500 rpm $\times 40$ minutes, $4^{\circ} \mathrm{C}$ ). The saliva was stored at $-20^{\circ} \mathrm{C}$ until the measurement. The time of collection was determined from 11:30 to $17: 30$ at which the value of cortisol stabilizes in consideration of the circadian variation of cortisol value [5] [6].

\subsection{The Measurement Method of Salivary Components}

SALIVARY IL-6 ELISA KIT (Salimetrics, USA) for the amount of IL-6, High Sensitivity SALIVARY CORTISOL ENZYME IMMUNOASSAY KIT (Salimetrics, USA) for the amount of cortisol, and Salivary Secretory IgA Indirect Enzyme Immuoassay kit (Salimetrics, USA) for the amount of SIgA were used for the measurement.

\subsection{The Measurement of Skin Color}

For seven female subjects, skin color was measured using Robo Skin Analyzer ${ }^{\circledR}$ (MM \& NiiC Co., Ltd.) besides collecting saliva at the monthly follow-ups from before the implant surgery and to six months after the surgery. Robo Skin Analyzer ${ }^{\circledR}$ is a measurement device for skin color with images taken by a CCD camera, and expresses the colors of skin as three attributions of hue, brightness, and intensity by measuring skin reflectivity [7] [8].

As the measurement conditions, after the subject washed her face to take her makeup off, three facial images (right $45^{\circ}$-front-left $45^{\circ}$ ) were taken with the built-in CCD camera keeping the distance from the object and the camera constant by fixing the forehead and the jaw in the box of Robo Skin Analyzer ${ }^{\circledR}$ which was blocked the surrounding environmental colors and kept the shooting condition constant.

(The correlation between the amount of IL-6, cortisol and SIgA and the facial color tones were discussed.)

\subsection{Statistical Analysis}

All the data are presented as mean \pm SEM. The data were analyzed by the Wilcoxon Rank Sum Test to evaluate the difference between pre-surgery and post-surgery. $\mathrm{p}$ values of $<0.05$ were defined as statistically significant.

\section{Results}

\subsection{The Change in Salivary Components}

The transition of the amount of IL-6 in saliva for six months was shown in Figure 1.

IL-6 increased rapidly after the treatment and kept high value repeating the increase and the decrease. However, it presented the comparable value as the one before the treatment at the measurement after six months when it changed to the final prosthesis. Comparing the changes in males to females, for males it remained high value after the treatment and presented the comparable value as the one before the treatment after six months when it changed to the final prosthesis (Figure 2(a)). For females on the other hand, it remained high value one month after the treatment, however, it transited at the comparable value as the one before the treatment after two months (Figure 2(b)).

The transition of the amount of cortisol in saliva for six months was shown in Figure 3. As for cortisol, it presented high value before the treatment, however it started decreasing after the treatment and it decreased significantly after six months when it changed to the final prosthesis. Comparing the changes in male and female, it presented the same transition patters and gender differences could not been observed (Figure 4). No correlation was shown between then although the correlation about the amount of IL-6 and cortisol in saliva was considered coefficient value at 0.2528 and $\mathrm{p}$ value at $\mathrm{p}<0.001$ (Figure 5).

As for SIgA, no change was shown during the six month treatment (Figure 6). 


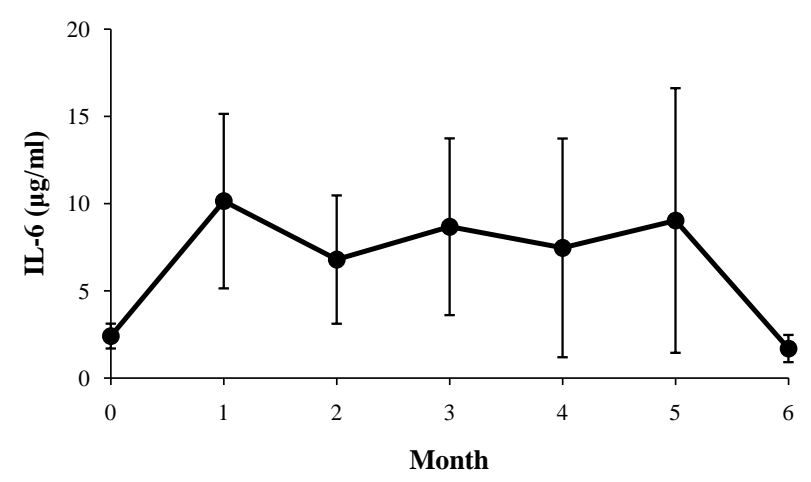

Figure 1. Temporal change of the salivary IL-6 levels (Mean \pm S.E, $n=14)$.

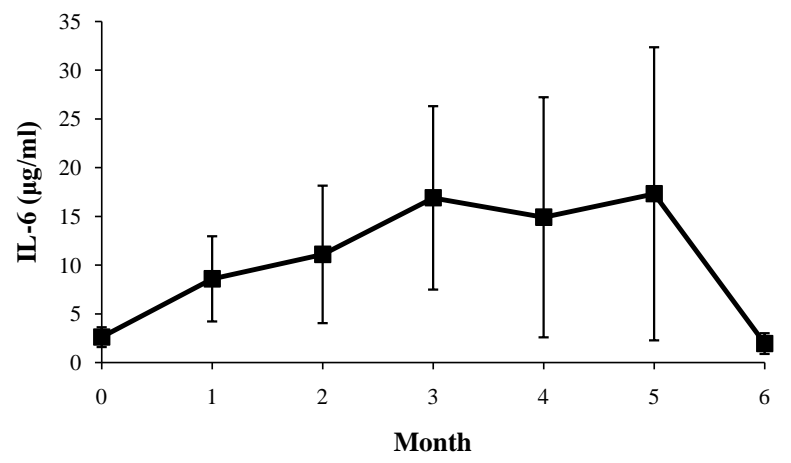

(a)

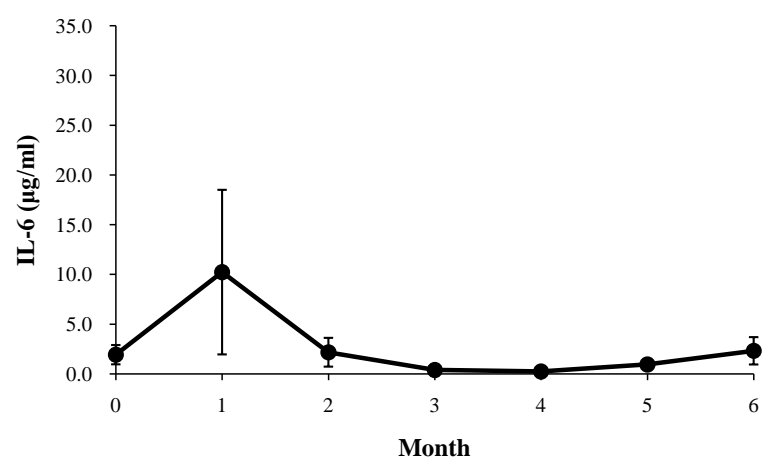

(b)

Figure 2. Temporal change of the salivary IL-6 levels (a) male (Mean \pm S.E, $n=7$ ); (b) female (Mean \pm S.E, $n=7$ ).

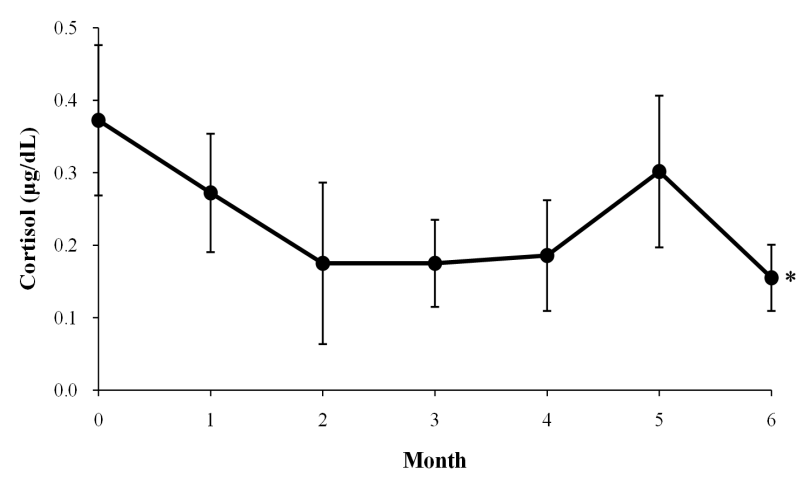

Figure 3. Temporal change of the salivary cortisol levels (Mean \pm S.E, $n=14$ ), ${ }^{*} \mathrm{p}<0.05$ vs. 0 .

\subsection{The Change in Skin Color}

The transition of three components of colors, hue, brightness, and intensity of seven female patients in six month was measured and its change was shown in Figure 7. The three components representing facial skin tones generally increased in ascension curve after the treatment. The hue increased significantly after six months compared to the one before the treatment.

\section{Discussion}

The clinical application of All-on-4 initiated in Japan from 2005, however, quite a lot of dental implant specialists inside and outside the country had objections to its innovation at the beginning. The goal of the oral implant 


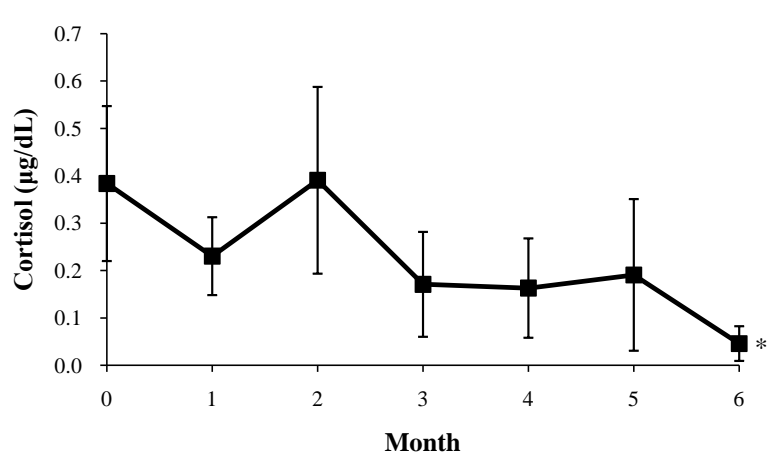

(a)

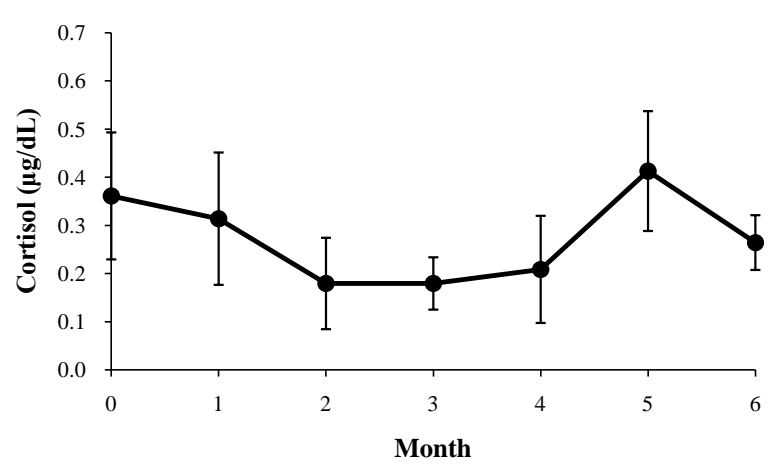

(b)

Figure 4. Temporal change of the salivary cortisol levels (a) male (Mean \pm S.E, $n=7$ ); (b) female (Mean \pm S.E, $n=7$ ), ${ }^{*} p<$ 0.05 vs. 0 .

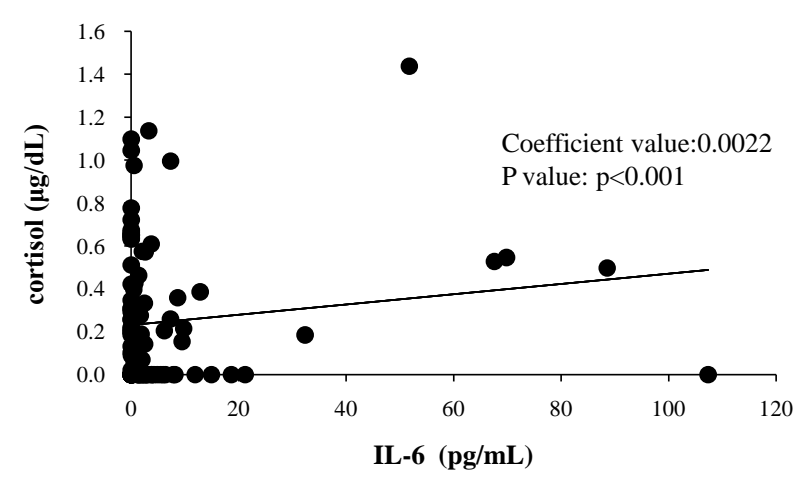

Figure 5. Correlation of salivary IL-6 and cortisol.

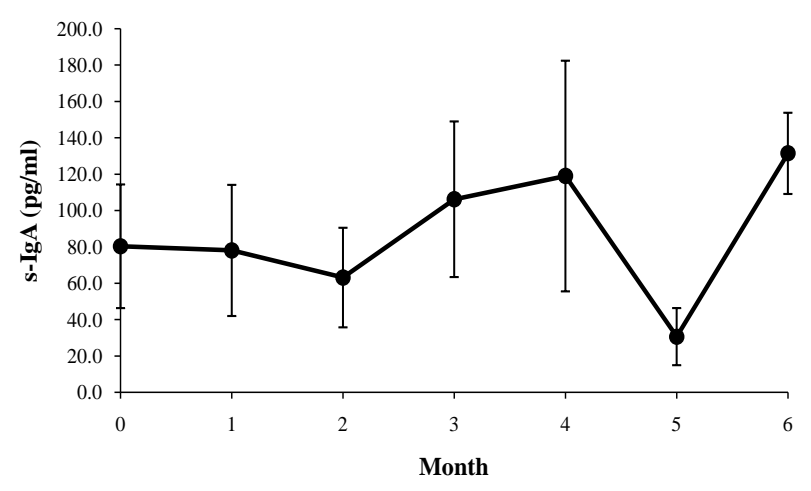

Figure 6. Temporal change of the salivary Ig-A (Mean \pm S.E, $\mathrm{n}=14)$.

technology before All-on-4 was just to replace the lost natural tooth with the artificial dental root as a substitute and to make it work. It is so to speak the biomimetics which is faithful to anatomical science [9]. Under the current dental implant situation, the success criteria are mostly about the connection of the bone and the implant fixture [10] [11], and the objective judgment criteria about obtaining masticatory movement and sensuousness which is the original goal for the occlusion function recovery are not required. The way to measure success and failure of the clinical implant is to examine the connection condition of the implant fixture and the bone, such as measurement of disturbance by the x-ray image diagnosis [12], the resonance frequency analysis [13], the measurement of the torque value and the Periotest [14] [15], and we only have to wait for the patient's complaint to notice the defect of the implant superstructure in the present circumstances. It is the beginning of this study to determine objectively if the immediate load dental implants which became close to us and the reconstruction of 


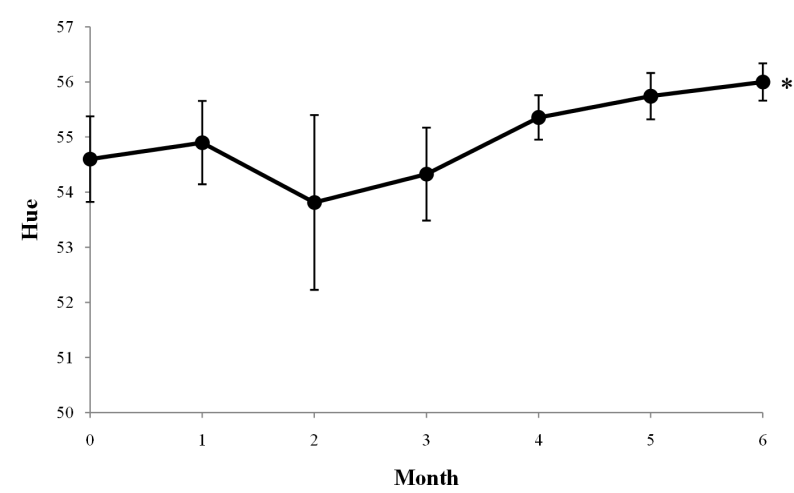

(a)

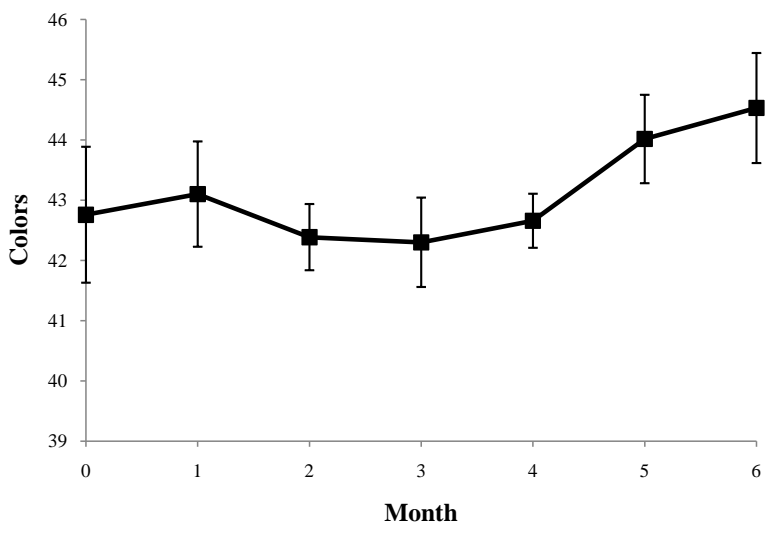

(b)

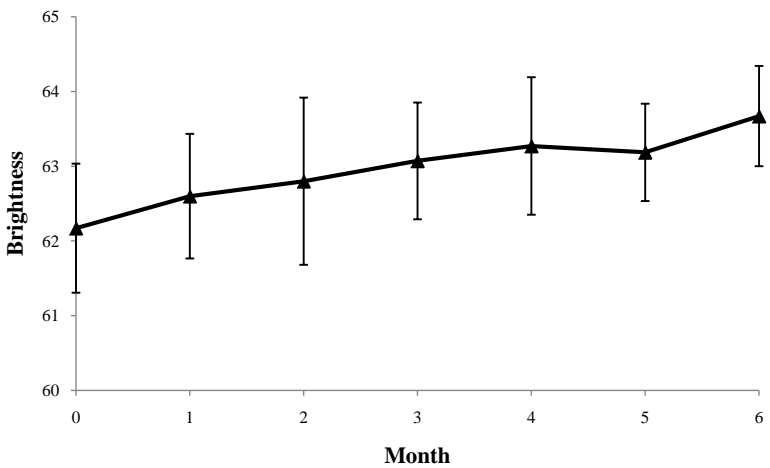

(c)

Figure 7. Temporal change of the skin-colored on the female patient after the implant operation (a) hue; (b) colors; (c) brightness (Mean \pm S.E, $n=7$ ), ${ }^{*}$ p $<0.05$ vs. 0 .

occlusion using the system really make the patients happy. For example, how the patient's mind and body who had replaced his or her teeth with artificial substitutes in a large ratio will change right after the implant surgery? What kinds of reactions will happen to the patient with the adjusting provisional bridge during the six months until the final prosthesis is completed? The patients' physical and psychological changes before and right after the implant surgery and during the six months of the treatment were examined through the correlation of the secretion concentration of the stress related substances in saliva.

In this study, the correlation between IL-6 in saliva and inflammation caused by surgical stress was proven as the amount of the measured value of IL-6 as inflammatory cytokine always increased remarkably after surgery and decreased to the comparative level of the one before surgery one month after the surgery although the difference of the values between the one before surgery and after surgery was concerned at first (Figure 1) [16][18].

The results of the present study demonstrated the correlation between IL-6 and surgical stress [19]-[22], or IL-6 and the inflammatory reaction caused by periodontitis [23]-[28]. The amount of IL-6 rapidly increased after surgery and remain high value almost all the time to the final prosthesis in six months, even though a little of the increase and the decrease was admitted in the middle, and the value became comparative to the one before surgery. The provisional bridge guides the mandibular position to the occlusion point where there are no tension on temporomandibular joint and masticatory muscle, and recovers occlusion at the central occlusion position where the occlusional surface between the upper and lower jaws connects at the maximum area and is considered as the most balanced jaw position in clinical dentistry [29].

On the other hand, as the performance needed for therapeutic dentures until it gets to the final prosthesis, the provisional bridge is recommended to be designed to avoid excessive occlusal pressure such as biting something hard. Differences among individuals reflected to the change of the graph in some degree, however the amount of IL-6 originated the process to infuse the implant body and the bone. It is reported that the implant stability de- 
creased for a while after surgery and recovered six months later by the research of the remodeling process of the implant fixture immediately loaded and the bone right after the placement to the molar area on the upper jaw [30], before surgery and every month after surgery using the resonance frequency analysis. The graph showing the implant stability during remodeling the early bone and the measurement graph showing the amount of IL-6 flipped vertically and were orbited symmetrically. This suggests that the amount of IL-6 is affected by the systematic changes around the implant.

The amount of cortisol did not indicate the extreme increase after surgery (Figure 3). The transition of the amount of cortisol basically tended to decrease gradually repeating the increase and the decrease from before the treatment to the final prosthesis transition in 6 months. Cortisol is secreted in response to the stress which is also the physical and psychological events causing distortion to homeostasis as a mechanism to keep the physiological situation in a body constant [31]. As both of stress caused by the physical and the psychological events stimulates ACTH (andreno-corticotorpic hormone) from hypothalamic-anterior pituitary and secretes cortisol from adrenal cortex [32], it is able to figure out whether the person is stressed or not by measuring cortisol [33]-[35]. As for the transition of cortisol, a patient's unique significant change of the amount of cortisol was observed as well as the change of IL-6, and the rapid shift of the increase and the decrease was observed at the different timing from IL-6. In reviewing medical records about the events happened to each patient at the observation point of the increase of the amount of cortisol, the patients complained about the adoption of the provisional bridge in their months in most of the situation, and it found out that the provisional bridge form was adjusted meticulously or repaired to fit in their mouths at the chair-side on demand from the patients in each case. As cortisol is the biomarker related to stress, it seemed that the patients removed stress associated with edentulous gradually, not right after the surgery when their occlusion was recovered. In particular, it seems that the process of the fusion of the implant fixture and the bone getting smooth and the process of the treatment that the provisional bridge form gradually got refined and adjusted in the patients' mouths on demand from patients at the monthly clinic visits was expected. In general, the graph gently decreasing formed a specific peak at the point of five months and it showed the increase of the amount of cortisol, however, the increase of the amount of cortisol was observed for two patients at this time of saliva measurement, and the amount of cortisol constantly transited with high value for one patient from before the treatment and during the treatment. This was the case that the only two mandibular molar teeth on the right side were compensated with the implants and the untreated remaining teeth were mixed although All-on-4 was applied to the upper jaw, and the crown prosthesis on bicuspid tooth on the right side which was the implant adjacent tooth was taken off at the time of this measurement. The body of the provisional bridge was fractured for the other patient. For each case, the increased amount of cortisol decreased at the next month's measurement after duplicating the prosthesis and repairing the provisional bridge. It was expected that there would be the positive correlation between the amount of IL-6 and cortisol as both of IL-6 and cortisol increased in respond to some events, however the correlation between the two was not observed. Moreover IL-6 increased when a systematic change which caused the damages to the implant fixture and the fusion of the implant fixture around the gum and the bones like preventing to promote healing was observed even though the patients did not notice any symptoms. Cortisol increased when the patient noticed discomfort and mentioned any events under stress on his or her medical record.

In this study, IL-6 indicated that systemic abnormalities about the jawbones which built the basic part to support artificially reconstructed occlusion, implant fixture, and the surrounding tissue, and cortisol could be considered as something to alert the mechanical problems about the implant superstructure which played a role of a fixed over denture with implant fixtures and screws. The information is extracted from two aspects, periodontological and prosthesis by using both for the measurement. By evaluating this data and the implant stability obtained from clinical x-ray images or by resonance frequency analysis, there is a possibility that it will help modifying the provisional bridge more accurately, designing the final prosthesis and for the post operational management. Moreover, as the amount of IL-6 and cortisol both indicated the lowest value in six months, the clinically recommended guide that the timing to transit to the final prosthesis was six months after the implant replacement when the bone fusion stabilized was confirmed.

For the female subjects, it was measured by using Robo Skin Analyzer ${ }^{\circledR}$ how it affected to the skin condition by recovering the occlusional function. The anti-aging theory that recovering occlusion can gain their youth is commonly known. The measuring intention here, however is to discuss how the occlusion recovery spills over to the rejuvenation effect not only around the mouth and the lips but also on physical appearance of a whole body by measuring freshness and moisture of women's skin condition with the image analysis and by comparing 
the amount of IL-6 as inflammatory cytokine, cortisol as a stress related biomarker, and SIgA related to immune competence. Three components of colors, hue, brightness, and intensity, were measured as an indicator by Robo Skin Analyzer ${ }^{\circledR}$. All of hue, brightness and intensity gently increased in six months after surgery and the improvement of skin tone color was observed. In conclusion, statically, the provisional restoration recovered maxillary and mandibular occlusion at first, set the jaw position to the central occlusion point, and counterbalanced and stabilized the mandibular position by masseter muscle, temporal muscle and internal and external pterygoid muscle, which organized the back and forth and lateral movement on lower jaw, keeping moderate load. It also maintained moderate stress on the mimic muscle group gathered in a lip and a mouth, consists of orbicularis oris muscle, cheek muscle, laughing muscle, mentalis muscle, musculus levator anguli oris, depressor anguli oris muscle, greater zygomatic muscle, lesser zygomatic muscle, elevator muscle of upper lip, depressor muscle of lower lip, etc., by compensating the lost dentition and alveolar part artificially. Dynamically, moreover, the movement of the masseter muscle group promoted as the masticatory function became active. As for the effect of esthetic recovery, the actions with positive expression, such as smiling with teeth, became diverse. Therefore it promoted the movement of the mimic muscle group which spread to the whole face, such as frontalis muscle, orbicularis oculi muscle and corrugator muscle leading from the mimic muscle of a mouth, and stimulated lymphatic circulation. It is considered that these results were reflected to the skin condition. Furthermore, it is expected that daily stress will be relived if the irritation on eating and the frustration with the physical appearance are removed. However regarding the correlation of the graph of the amount of cortisol for the subjected seven female patients, their facial hue, brightness, and intensity increased in contradiction to the amount of cortisol gradually decreased every time at the follow-ups after surgery. The decrease of the amount of cortisol and the improvement of their facial color tone observed six months after the surgery suggested the possibility that the recovery of the occlusion function by the immediate load dental implants did not only make the patients expressive for their physical appearance health but also brought their skin firmness and moisture by making their "feeling" balanced and affected deeply to the physical and psychological health promotion through the acquisition of masticatory function and the aesthetic recovery.

It is a purpose to measure how an effect of the oral rehabilitation applied immediate loading implant system affects the body as for this study, but, about the physical influence except the oral area, does not lecture in particular. The consideration about the influence that the physical factor except the oral area caused by health condition and the psychology situation of the subject and a lifestyle affects measurement data is a future research.

Facing an aging society, the demand for the treatment applying the immediate load dental implants will grow. Along with it, however, the situations that patients who take the dental implant treatment would visit other branches or they would need a long-term hospitalization are concerned as well. Originally strict and periodic maintenances by professionals are necessary to maintain the restructured occlusion with the implant treatment [36]. However, if stress reactant materials which can be measured by the non-invasive and simple saliva collection can be applied to the diagnosis as the biomarker [37], it may be contributory to find out oral functional problems in the future medical front.

Moreover, the decrease of stress reactant hormones and the improvement of skin color tones obtained from the analysis by Robo Skin Analyzer ${ }^{\circledR}$ indicated that the occlusion recovery released the patients from physical and psychological stress, such as the decrease of masticatory function and aesthetical complex, and enriched their "feeling". It is expected that the restructure of occlusion by the immediate load dental implants brings the patients smile back and recuperates their sociability and positive attitude, and enhances their quality of life.

\section{References}

[1] Maló, P., Rangert, B. and Nobre, M. (2003) “All-on-Four” Immediate-Function Concept with Brånemark System Implants for Completely Edentulous Mandibles: A Retrospective Clinical Study. Clinical Implant Dentistry and Related Research, 5, 2-9.

[2] Tarnow, D.P., Emtiaz, S. and Classi, A. (1997) Immediate Loading of Threaded Implants at Stage 1 Surgery in Edentulous Arches: Ten Consecutive Case Reports with 1- to 5-Year Data. The International Journal of Oral \& Maxillofacial Implant, 12, 319-24.

[3] Garg, A.K. (2007) Osstell Mentor: Measuring Dental Implant Stability at Placement, before Loading, and after Loading. Dent Implantol Update, 18, 49-53.

[4] Kim, H., Kashiwagi, K. and Kawazoe, T. (2009) Measurement Errors for the Primary Stability of Implants Using a Wireless Resonance Frequency Analyzer. The Journal of the Osaka Odontological Society, 72, 69-76. 
[5] Hucklebridge, F., Clow, A. and Evans, P. (1998) The Relationship between Salivary Secretory Immunoglobulin A and Cortisol: Neuroendocrine Response to Awakening and the Diurnal Cycle. International Journal of Psychophysiology, 31, 69-76. http://dx.doi.org/10.1016/S0167-8760(98)00042-7

[6] Knutsson, U., Dahlgren, J., Marcus, C., Rosberg, S., Brönnegård, M., Stierna, P. and Albertsson-Wikland, K. (1997) Circadian Cortisol Rhythms in Healthy Boys and Girls: Relationship with Age, Growth, Body Composition, and Pubertal Development. The Journal of Clinical Endocrinology \& Metabolism, 82, 536-403.

[7] Akimoto, M., Miyazaki, M., Lee, H.-H., Nishimura, T., Tamura, M. and Miyakawa, M. (2009) Using Fuzzy Reasoning to Support System of Diagnosis of the Skin Disease. Bioimages, 17, 11-20.

[8] Takiwaki, H. (1998) Measurement of Skin Color Using Reflectance Instruments and Image Analysis of the Skin. Cosmetic Stage. The Journal of Medical Investigation, 44, 121-126.

[9] Misch, C.E. and Degidi, M. (2003) Five-Year Prospective Study of Immediate Early Loading of Fixed Prostheses in Completely Edentulous Jaws with a Bone Quality-Based Implant System. Clinical Implant Dentistry and Related Research, 5, 17-28. http://dx.doi.org/10.1111/j.1708-8208.2003.tb00178.x

[10] Albrektsson, T. and Sennerby, L. (1991) State of the Art in Oral Implants. Journal of Clinical Periodontology, 18, 474-481. http://dx.doi.org/10.1111/j.1600-051X.1991.tb02319.x

[11] Zarb, G.A. and Albrektsson, T. (1998) Consensus Report: Towards Optimized Treatment Outcomes for Dental Implants. Journal of Prosthetic Dentistry, 80, 641.

[12] Lindh, C., Petersson, A. and Rohlin, M. (1996) Assessment of the Trabecular Pattern before Endosseous Implant Treatment: Diagnostic Outcome of Periapical Radiography in the Mandible. Oral Surgery, Oral Medicine, Oral Pathology, Oral Radiology, and Endodontology, 82, 335-343. http://dx.doi.org/10.1016/S1079-2104(96)80363-5

[13] Meredith, N., Alleyne, D. and Cawley, P. (1996) Quantitative Determination of the Stability of the Implant-Tissue Interface Using Resonance Frequency Analysis. Clinical Oral Implants Reseach, 7, 261-267. http://dx.doi.org/10.1034/j.1600-0501.1996.070308.x

[14] Johansson, P. and Strid, K.G. (1994) Assessment of Bone Quality from Placement Resistance during Implant Surgery. The International Journal of Oral \& Maxillofacial Implant, 9, 279-288.

[15] Olivé, J. and Aparicio, C. (1990) Periotest Method as a Measure of Osseointegrated Oral Implant Stability. The International Journal of Oral \& Maxillofacial Implant, 5, 390-400

[16] Kishimoto, T. (2005) Interleukin-6: From Basic Science to Medicine-40 Years in Immunology. Annual Review of Immunology, 23, 1-21. http://dx.doi.org/10.1146/annurev.immunol.23.021704.115806

[17] Hirano, T., Akira, S., Taga, T. and Kishimoto, T. (1990) Biological and Clinical Aspects of Interleukin 6. Immunol Today, 11, 443-449. http://dx.doi.org/10.1016/0167-5699(90)90173-7

[18] Ohzato, H., Yoshizaki, K., Nishimoto, N., Ogata, A., Tagoh, H., Monden, M., Gotoh, M., Kishimoto, T. and Mori, T. (1992) Interleukin-6 as a New Indicator of Inflammatory Status: Detection of Serum Levels of Interleukin-6 and C-Reactive Protein after Surgery. Surgery, 111, 201-209.

[19] Gabay, C. and Kushner, I. (1999) Acute-Phase Proteins and Other Systemic Responses to Inflammation. The New England of Journal Medicine, 340, 448-454.

[20] Douglas, R.G. and Shaw, J.H. (1989) Metabolic Response to Sepsis and Trauma. British Journal of Surgery, 76, 115122.

[21] Levine, S.J., Larivée, P., Logun, C., Angus, C.W. and Shelhamer, J.H. (1993) Corticosteroids Differentially Regulate Secretion of IL-6, IL-8, and G-CSF by a Human Bronchial Epithelial Cell Line. American Journal of Physiology, 265, 360-368.

[22] Kato, M., Suzuki, H., Murakami, M., Akama, M., Matsukawa, S. and Hashimoto, Y. (1997) Elevated Plasma Levels of Interleukin-6, Interleukin-8, and Granulocyte Colony-Stimulating Factor during and after Major Abdominal Surgery. Journal of Clinical Anesthesia, 9, 293-298. http://dx.doi.org/10.1016/S0952-8180(97)00006-8

[23] Kamagata, Y., Miyasaka, N., Inoue, H., Hashimoto, J. and Iida, M. (1989) Cytokine Production in Inflamed Human Gingival Tissues-Interleukin-6. Journal of Japanese Society of Periodontology, 31, 843-848. http://dx.doi.org/10.2329/perio.31.843

[24] Sugano, N. (1992) Study of Interleukin-6 Expression in Periodontitis. Journal of Japanese Society of Periodontology, 34, 277-285. http://dx.doi.org/10.2329/perio.34.277

[25] Takahashi, K. (1992) Assessment of Interleukin-6 in the Progression of Disease. Journal of Japanese Society of Periodontology, 34, 286-300. http://dx.doi.org/10.2329/perio.34.286

[26] Takigawa, M. (1992) Study of Interleukin-6 Produced from Human Gingival Fibroblasts. Journal of Japanese Society of Periodontology, 34, 301-314. http://dx.doi.org/10.2329/perio.34.301

[27] Ito, H., Takata, T., Miyauchi, M., Ogawa, I. and Nikai, H. (1994) Immunohistochemical Localization of Interleu- 
kin- $1 \alpha$,Interleukin- $1 \beta$ and Interleukin-6 in Human Gingival Tissue. Journal of Japanese Society of Periodontology, 36, 545-551. http://dx.doi.org/10.2329/perio.36.545

[28] Yamaguchi, T. (2009) Enamel Protein Suppress Inflammatory Reaction. Journal of Japanese Society of Periodontology, 51, 38-50. http://dx.doi.org/10.2329/perio.51.038

[29] Hobo, S. (1984) A Kinematic Investigation of Mandibular Border Movement. Journal of Prosthetic Dentistry, 51, 642-646. http://dx.doi.org/10.1016/0022-3913(84)90409-8

[30] Glauser, R., Portmann, M., Ruhstaller, P., Lundgren, A.K., Hammerie, C.H. and Gottlow, J. (2001) Stability Measurements of Immediately Loaded Machined and Oxidized Implants in the Posterior Maxilla. A Comparative Clinical Study Using Response Frequency Analysis. Applied Osseointegration Research, 2, 27-29.

[31] Cannon, W.B. (1914) The Emergency Function of the Adrenal Medulla in Pain and the Major Emotions. American Journal of Physiology, 33, 356-372.

[32] Selye, H. (1936) A Syndrome Produced by Diverse Nocuous Agents. Nature, 138, 32. http://dx.doi.org/10.1038/138032a0

[33] Izawa, S. and Suzuki, K. (2007) The Comparison of Salivary Cortisol Immunoassay Kits: Correlations between Salivary and Plasma Cortisol Concentrations and Comparison of Immunoassay Methods. Japanese Journal of Complementary and Alternative Medicine, 4, 113-118. http://dx.doi.org/10.1625/jcam.4.113

[34] Ozawa, H. (2008) Neuronal Network for Regulation System of Stress, Feeding and Sexual Behavior. The Medical Association of Nippon Medical School, 4, 25-31. http://dx.doi.org/10.1272/manms.4.25

[35] tatsuo, M., Mitsunori, U., Hajime, I. and Masakazu, K. (2009) Stress Evaluation by the Stress Response Material in the Saliva and Effect by Bruxism. The Journal of Gifu dental Society, 36, 135-148.

[36] Mori, K. and Akiyoshi, M. (1967) Marginal Periodontitis: A Histological Study of Incipient Stage. Journal of Periodontology, 38, 45-52. http://dx.doi.org/10.1902/jop.1967.38.1.45

[37] Vining, R.F., McGinley, R.A., Maksvytis, J.J. and Ho, K.Y. (1983) Salivary Cortisol: A Better Measure of Adrenal Cortical Function than Serum Cortisol. Annals of Clinical Biochemistry, 20, 329-335.

http://dx.doi.org/10.1177/000456328302000601 